\title{
Comparison of Lumbopelvic Rhythm between Healthy Individuals and Lumbar Clinical Instability Patients
}

\section{Mohammad Pouretezad ${ }^{(\mathbb{D})}$, Milad Zarrin 2,*(i) , Reza Salehi ${ }^{3}$, Hossein Negahban $^{4}$, Mohammad Jafar Shaterzadeh Yazdi ${ }^{5}$, Mohammad Mehravar ${ }^{6}$}

${ }^{I}$ PhD in Physiotherapy, Musculoskeletal Rehabilitation Research Center, Ahvaz Jundishapur University of Medical Sciences, Ahvaz, Iran

${ }^{2}$ M.Sc Student in Physiotherapy, Student Research Committee, Ahvaz Jundishapur University of Medical Sciences, Ahvaz, Iran

${ }^{3}$ Associate Professor, Rehabilitation Research Center, Department of Rehabilitation Management, School of Rehabilitation Sciences, Iran University of Medical Sciences, Tehran, Iran

${ }^{4}$ Professor, Orthopedic Research Center Department of Physiotherapy, School of Paramedical Sciences, Mashhad University of Medical Sciences, Mashhad, Iran

${ }_{5}^{5}$ Associate Professor, Musculoskeletal Rehabilitation Research Center, Ahvaz Jundishapur University of Medical Sciences, Ahvaz, Iran

${ }^{6}$ Instructor, Musculoskeletal Rehabilitation Research Center, Ahvaz Jundishapur University of Medical Sciences, Ahvaz, Iran

* Corresponding Author: Milad Zarrin, Student Research Committee, Ahvaz Jundishapur University of Medical Sciences, Ahvaz, Iran. Email: miladzarrin.pt1995@gmail.com

\begin{tabular}{|c|c|}
\hline & Abstract \\
\hline $\begin{array}{l}\text { Received: } 23.04 .2021 \\
\text { Accepted: } 02.08 .2021\end{array}$ & \multirow{3}{*}{$\begin{array}{l}\text { Background and Objective: The lumbopelvic rhythm is the coordinated } \\
\text { movement of the lumbar spine and hip during trunk flexion and return. It is } \\
\text { recognized as a clinical indicator of low back pain (LBP); nonetheless, the } \\
\text { reported patterns of lumbopelvic rhythm in patients with LBP are } \\
\text { inconsistent. The investigation of more homogeneous subgroups of patients } \\
\text { with LBP is essential to clarify the lumbopelvic rhythm patterns. Therefore, } \\
\text { the present study aimed to compare lumbopelvic rhythm between healthy } \\
\text { individuals and patients with lumbar clinical instability. } \\
\text { Materials and Methods: A total of } 44 \text { subjects ( } 22 \text { healthy cases and } 22 \\
\text { lumbar clinical instability (LCI) patients) participated in the present study. } \\
\text { The kinematic parameters during the trunk flexion and return task were } \\
\text { recorded using a Qualisys motion capture system. Hip flexion angle, lumbar } \\
\text { flexion angle, and lumbopelvic rhythm were statistically analyzed in every } \\
25 \% \text { of the flexion and return phase of flexion. Data were analyzed using } \\
\text { Kolmogorov-Smirnov, Chi-square, and independent t-test. } \\
\text { Results: In the second quarter of trunk flexion, the flexion angle of the } \\
\text { lumbar spine was larger in the lumbar clinical instability group, as compared } \\
\text { to that in the control group (P=0.016). Furthermore, the hip flexion angle } \\
\text { was smaller in the lumbar clinical instability group, in comparison with that } \\
\text { in the control group (P=0.011). } \\
\text { Conclusion: These results show that the lumbopelvic rhythms are different } \\
\text { among healthy subjects and patients with lumbar clinical instability. } \\
\text { Keywords: Biomechanics, Kinematic, Low Back Pain }\end{array}$} \\
\hline $\begin{array}{l}\text { How to Cite this Article: } \\
\text { Pouretezad M, Zarrin M, Salehi } \\
\text { R, Negahban H, Shaterzadeh } \\
\text { Yazdi MJ, Mehravar M. } \\
\text { Comparison of Lumbopelvic } \\
\text { Rhythm between Healthy } \\
\text { Individuals and Lumbar Clinical } \\
\text { Instability Patients. Avicenna J } \\
\text { Clin Med. 2021; 28(2): } 95-103 \text {. } \\
\text { DOI: } 10.52547 / \text { ajcm.28.2.95 }\end{array}$ & \\
\hline & \\
\hline
\end{tabular}




\title{
مقايسه ريتم كمرى-الكنى افراد سالم و بيماران مبتلا به بىثباتى بالينى ستون فقرات كمرى
}

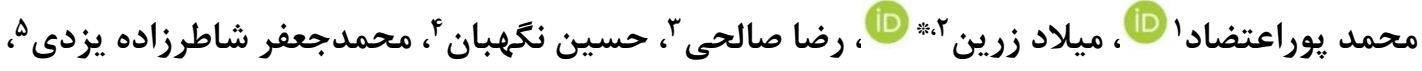 \\ محمد مهر آور
}

' دكترى تخصصى فيزيوترايى، مركز تحقيقات توانبخشى اسكلتى-عضلانى، دانشكاه علوم يزشكى جندى شايور اهواز، اهواز، ايران

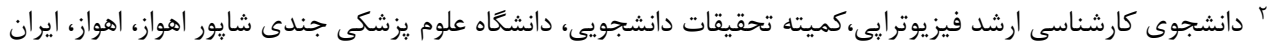

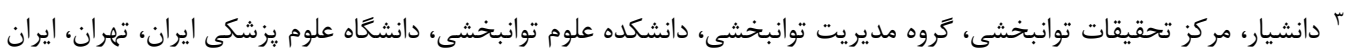

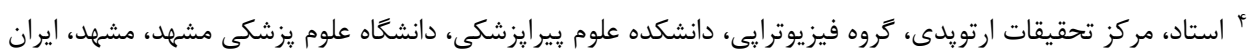

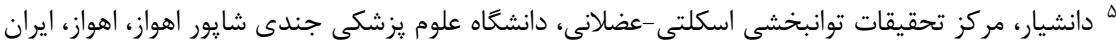

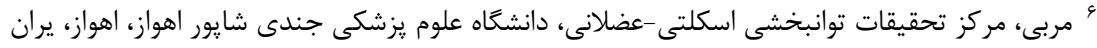
* نويسنده مسئول: ميلاد زرين، كميته تحقيقات دانشجويى، دانشكاه علوم بزشكى جندى شإيور اهواز، اهواز، ايران. ايميل: miladzarrin.pt1995@gmail.com

\begin{tabular}{|c|c|}
\hline 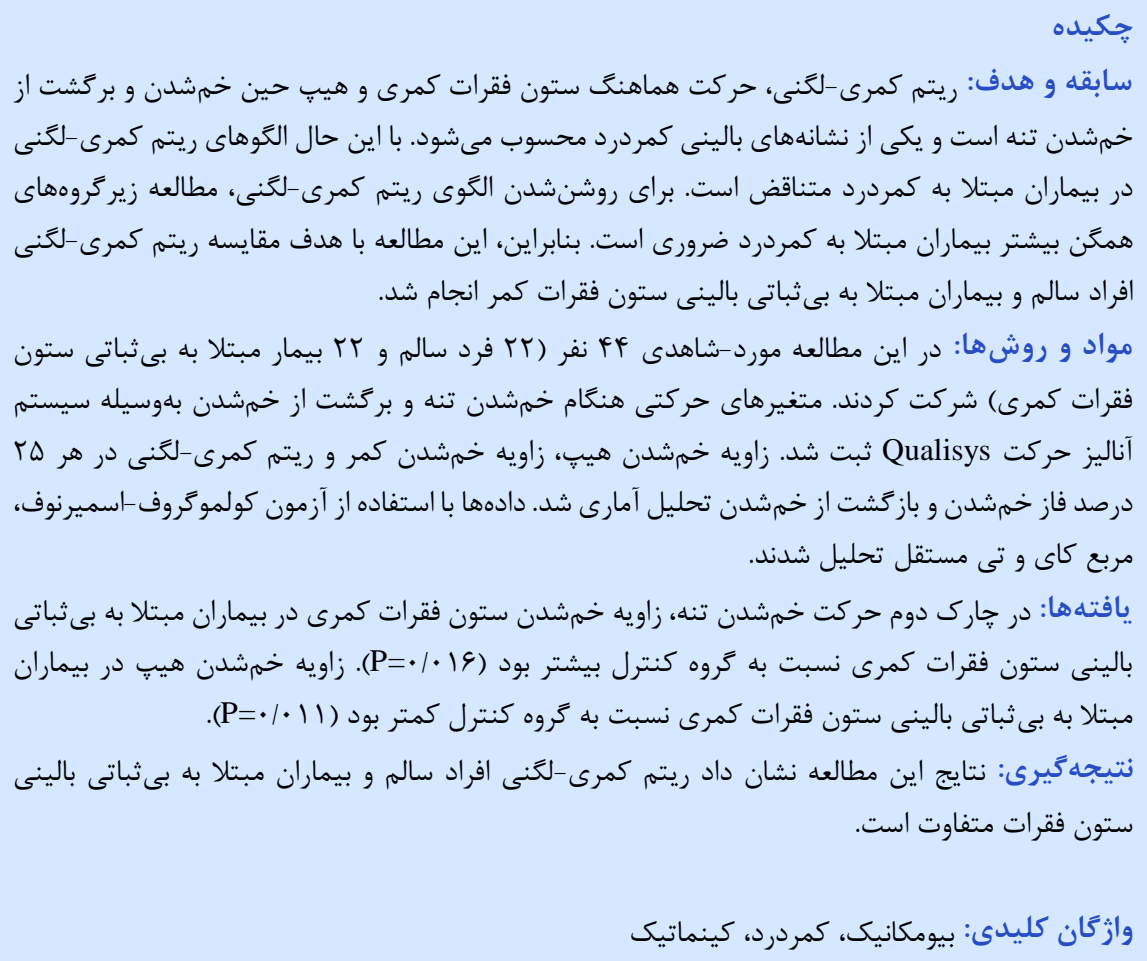 & 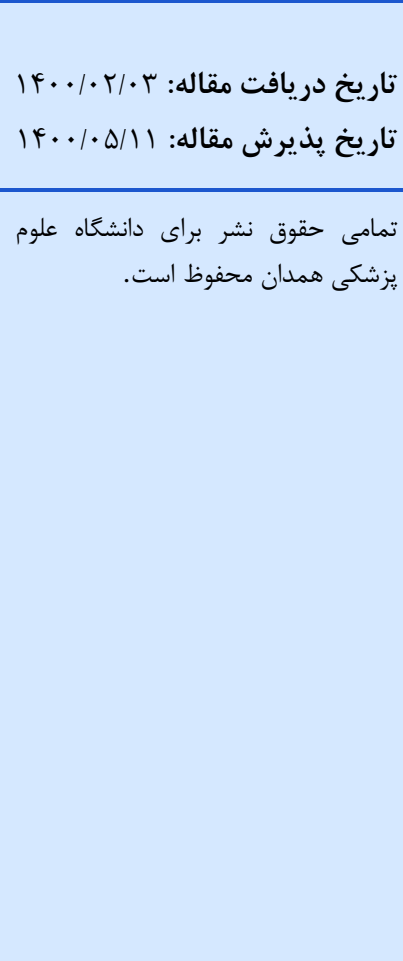 \\
\hline
\end{tabular}

خاصى ندارند و تحت عنوان كمردردهاى غيراختصاصى طبقهبندى مىشوند [ـ]، درحالى كه محققان متعددى معتقدند

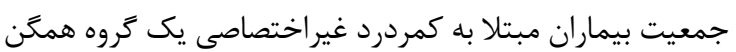

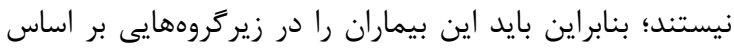

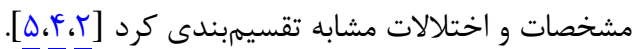

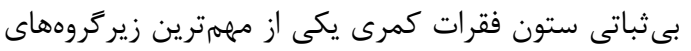

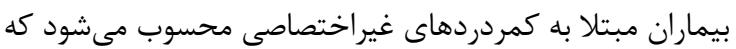

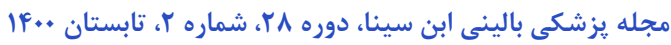

كمردرد يكى از شايع ترين اختلالات ماسكولواسكلتال

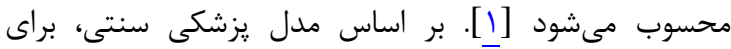

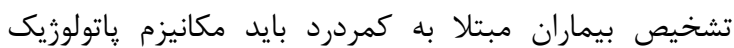
زمينهاى آن تعيين شود. اين در حالى است كه در بيشتر بيماران

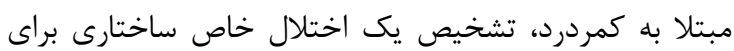

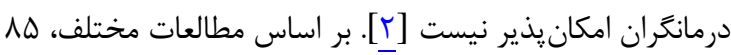

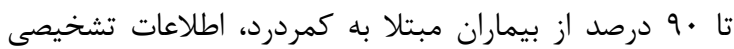


اختلال در ثبات موضعى ستون فقرات مىشود و از اين طريق

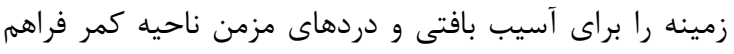

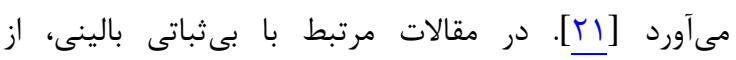

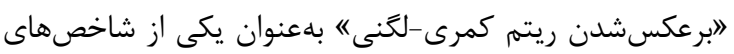

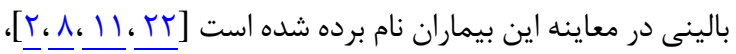

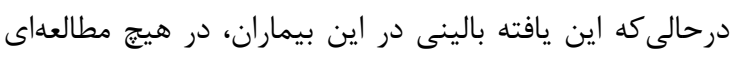

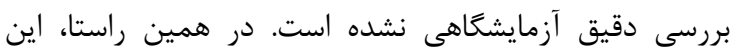

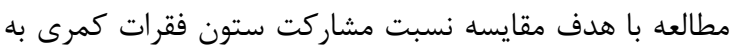

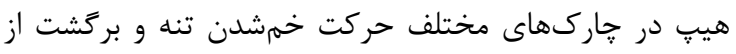

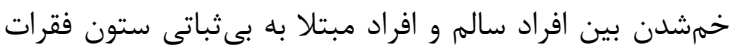

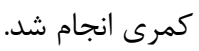

\section{مواد و روشها}

جامعه هدف در اين مطالعه مورد-شاهدى، بيماران مبتلا به

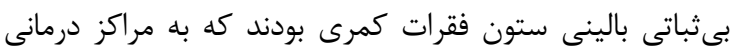

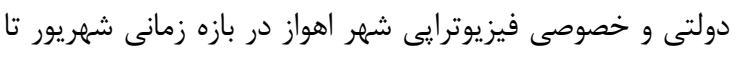

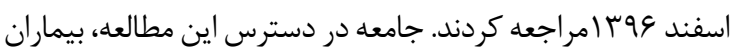

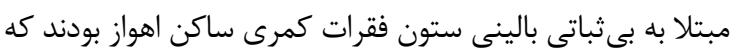

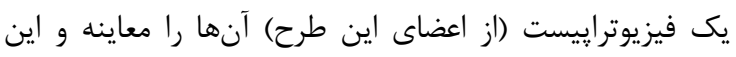

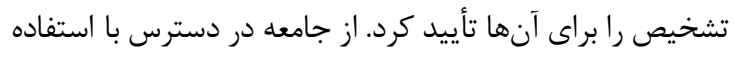

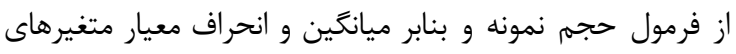

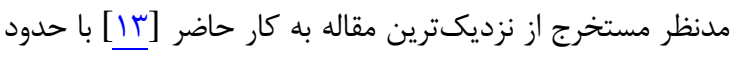

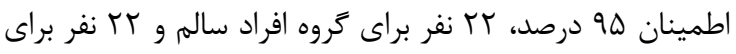

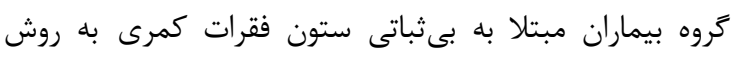

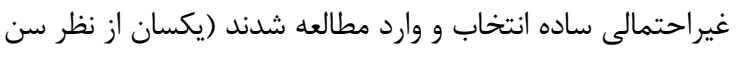

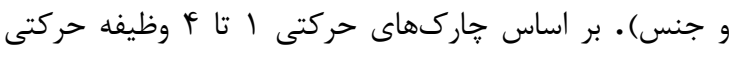

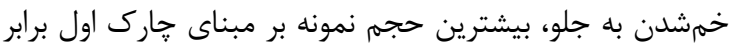

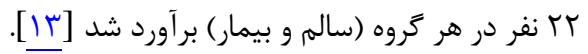

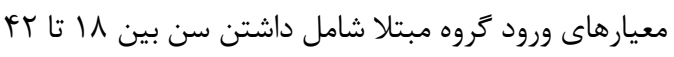

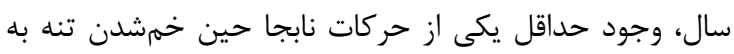

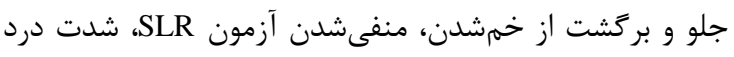

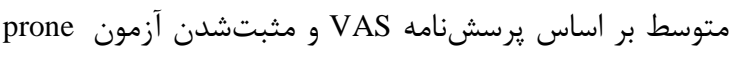
instability

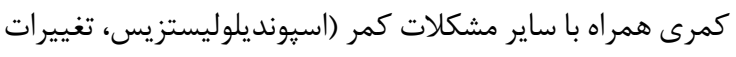

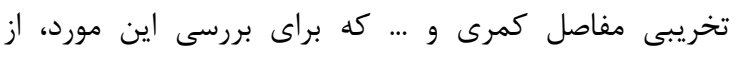
راديوگرافهاى لترال و قدامى-خلفى كمر استى استفاده شد)، سابقه

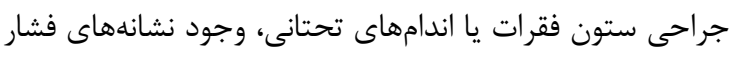

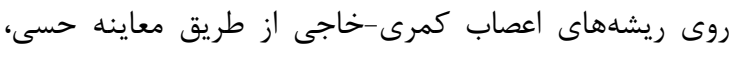

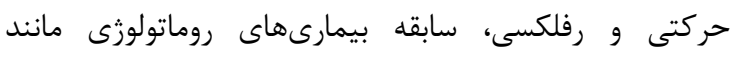

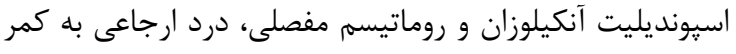

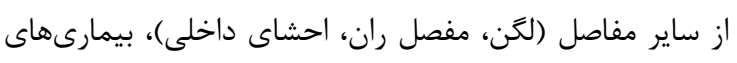

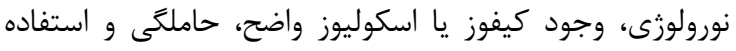

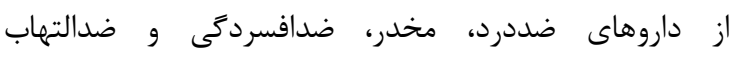

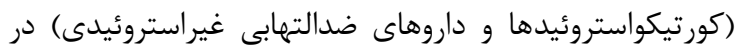

Panjabi

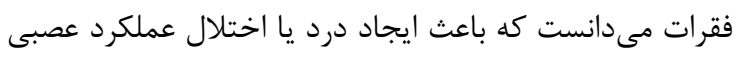

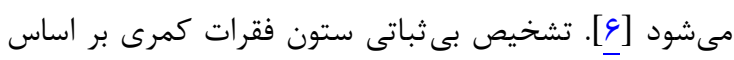

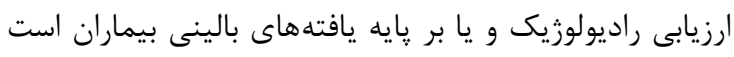

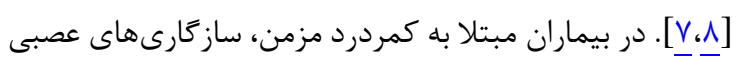

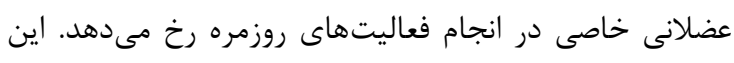

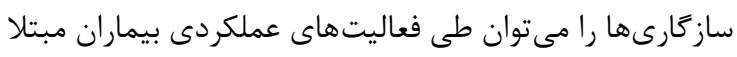

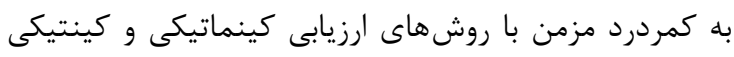

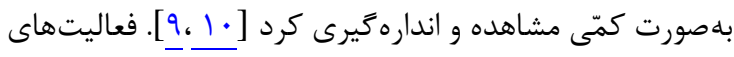

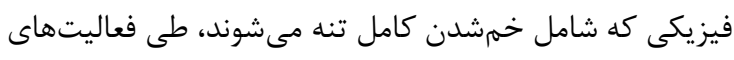

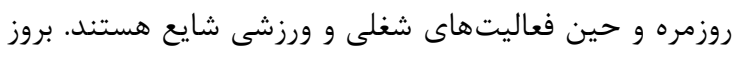

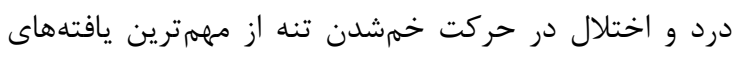

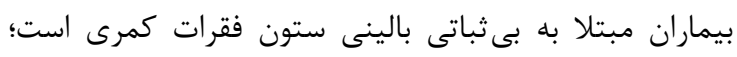

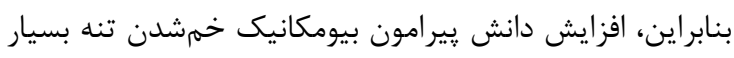

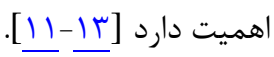

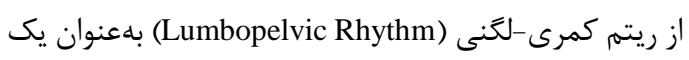

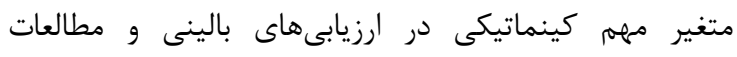

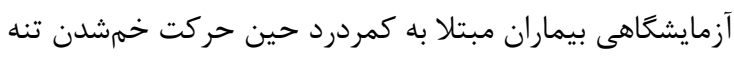

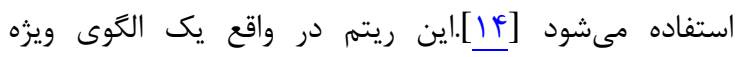

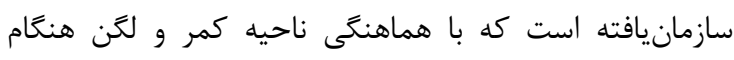

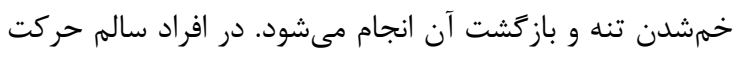

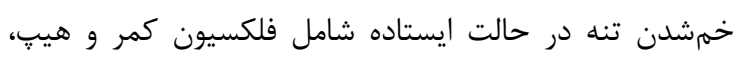

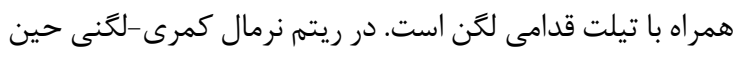

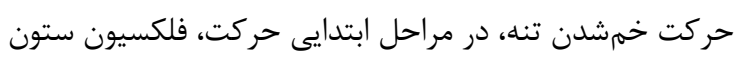

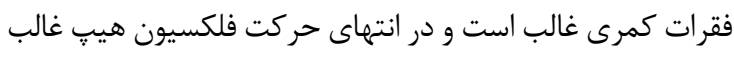

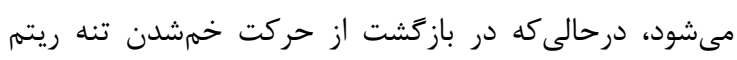

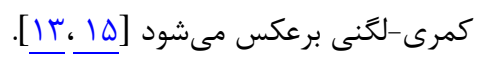

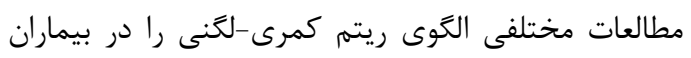

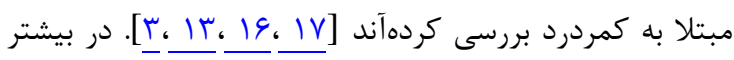

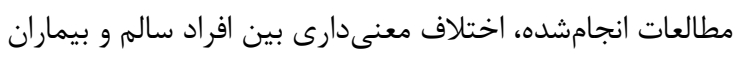

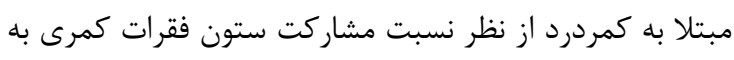

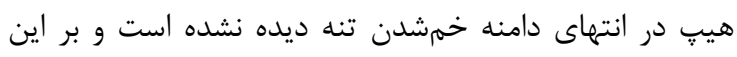

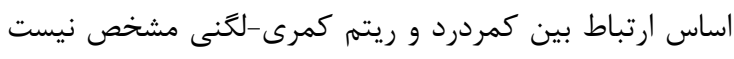

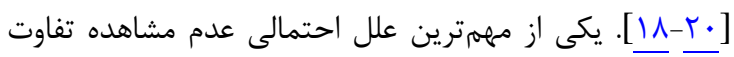

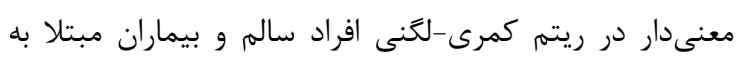

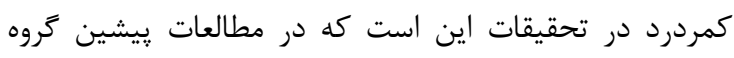

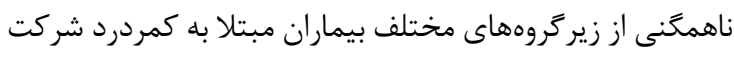

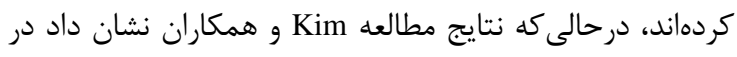

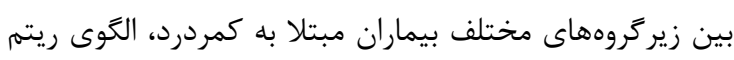

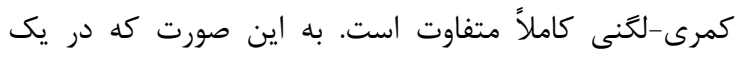

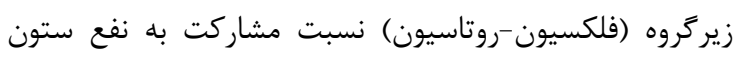

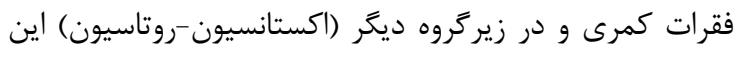

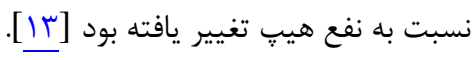
نقص در كنترل ديناميك مجموعه كمرى-لكنى باعث 
بس از نصب نشانكَرها، از فرد خواسته شد در حالت ايستادن

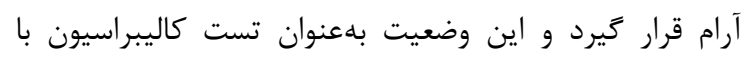
دوربينهاى مادون قرمز ثبت مىشد. همه تكرارها با نرمافز إزارسه بعدى Qualisys Medical AB, ) Qualisys Track Manager يردازش شدند. اطلاعات كينماتيكى (Sweden, Gothenburg ثبتشده با فيلتر مرتبه جهار دوطرفه Butter-worth فيلتر شدند.

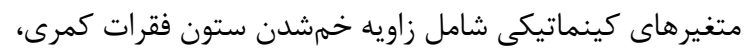

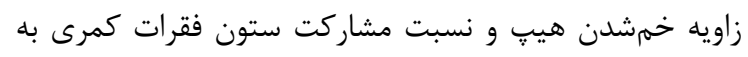

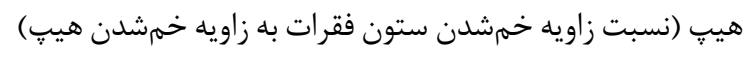

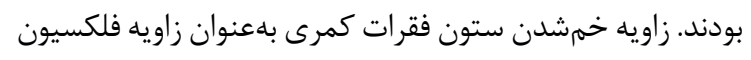

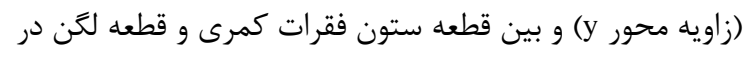

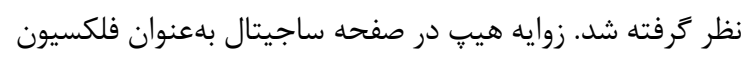

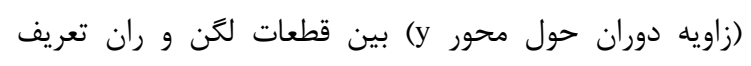

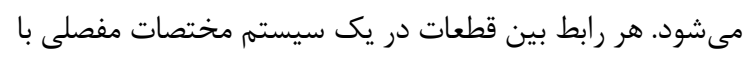

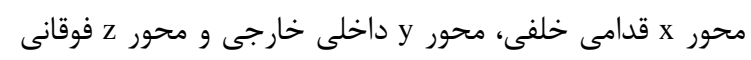

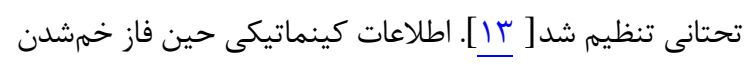

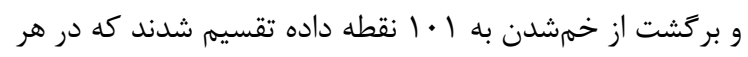

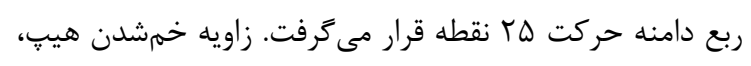

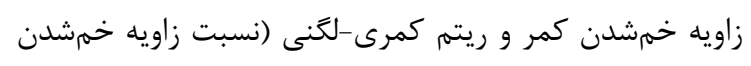

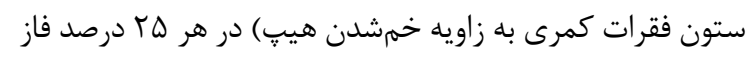

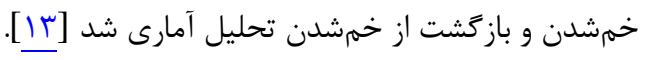

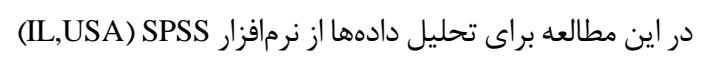

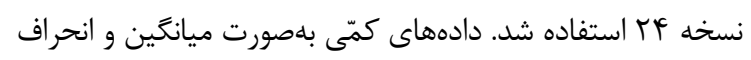

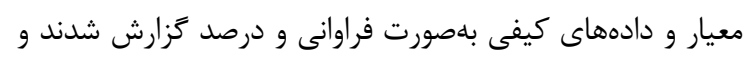

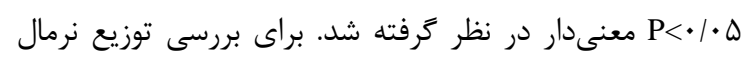

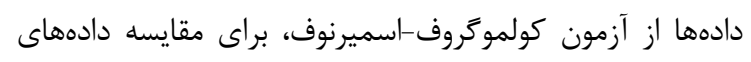

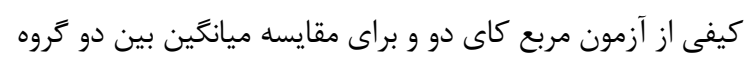
مورد و شاهد از آزمون تى مستقل استفاده شد.

\section{يافته ها}

بر اساس نتايج اين مطالعه، هيج تفاوت معنى داري، دارى در

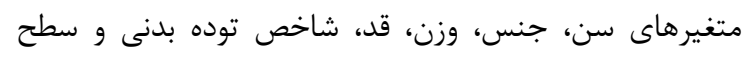

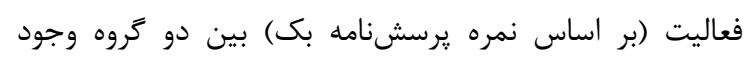

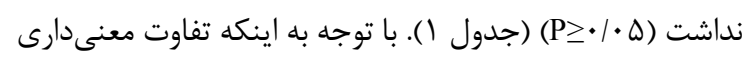

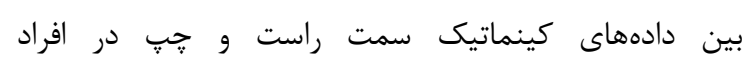

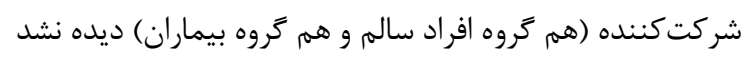

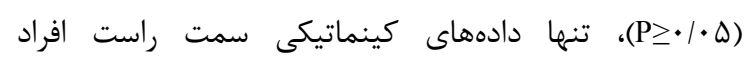

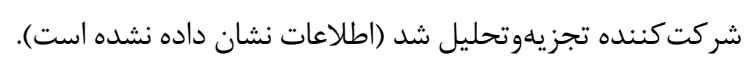

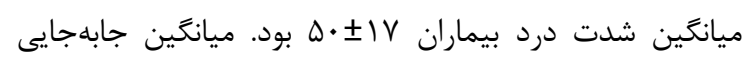

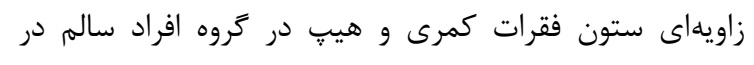

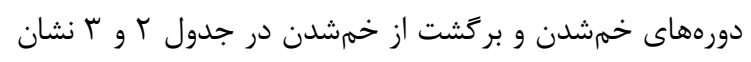

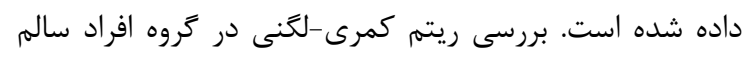

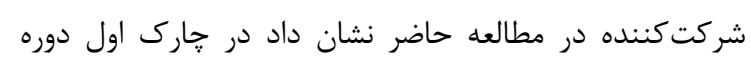

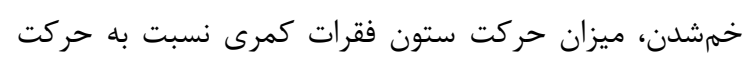

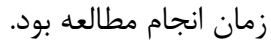

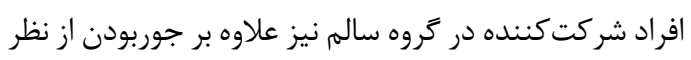

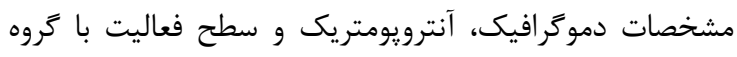

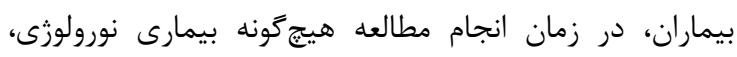

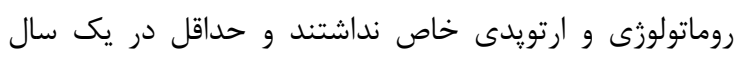

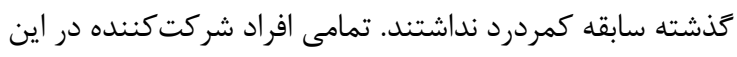

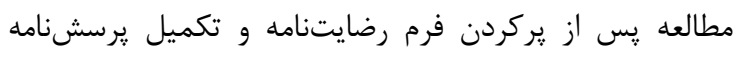
عمومى وارد تحقيق شدند.

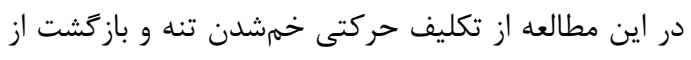

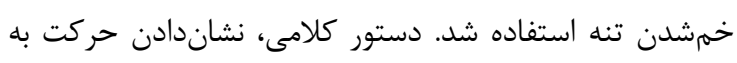

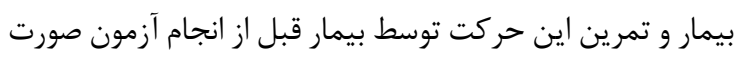

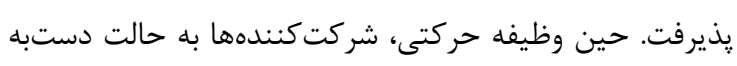

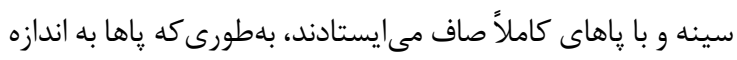

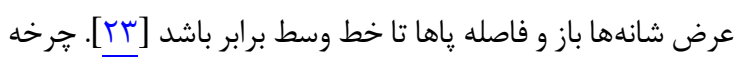

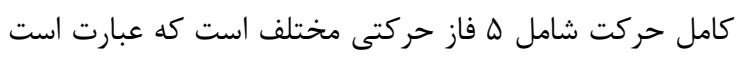

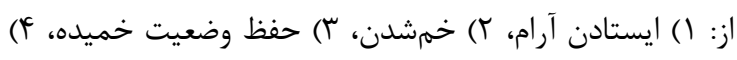

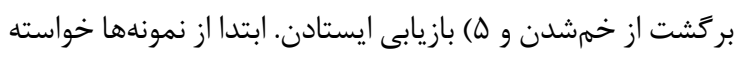

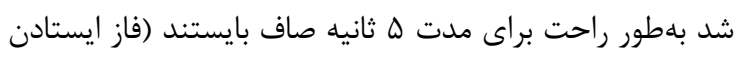

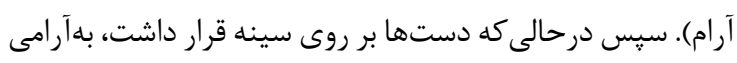

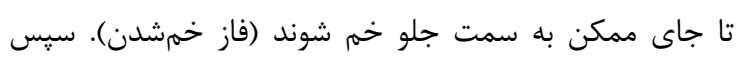

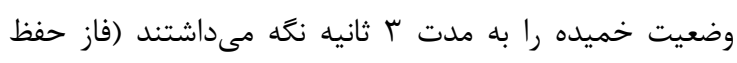

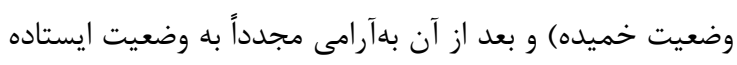

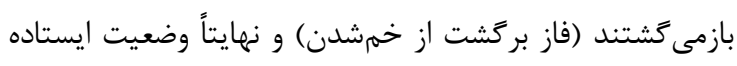

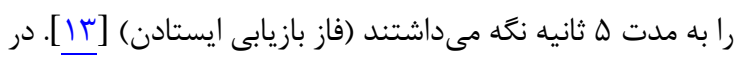

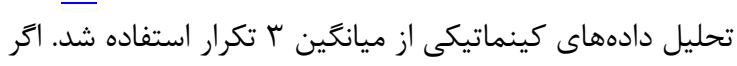

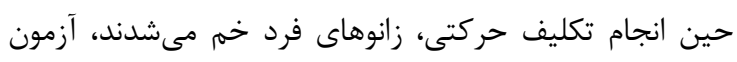
تكرار مىشد. در اين مطالعه از سيستم سه بعدى Qualisys Motion (Qualisys Medical AB, Sweden, Gothenburg) Capture شامل V دوربين مادون قرمز براى ثبت دادههاى كينماتيكى

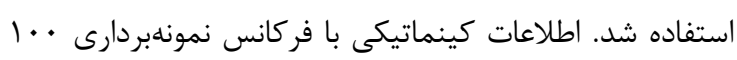

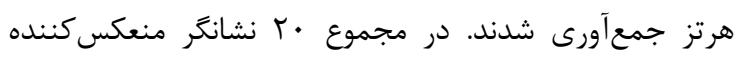

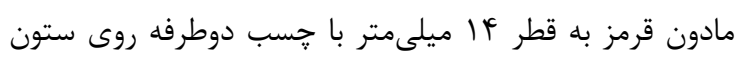

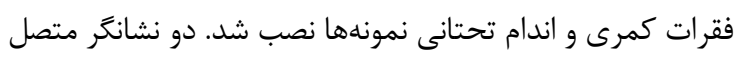

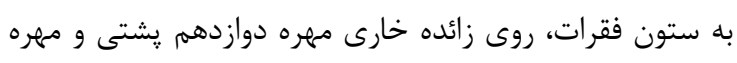

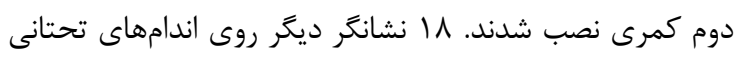

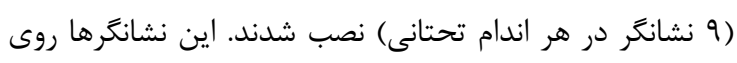

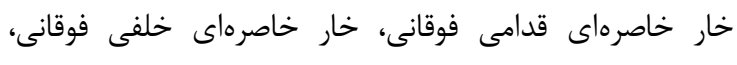

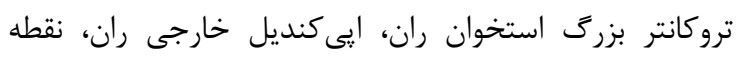

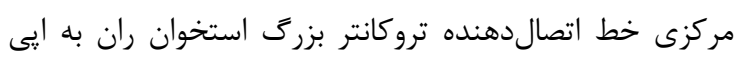

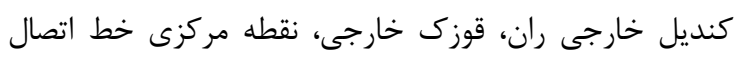

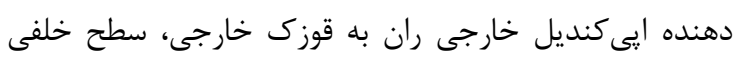

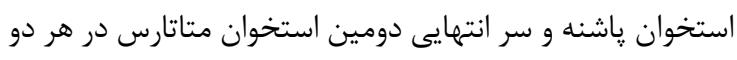

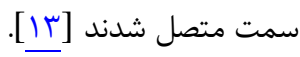


جدول ا: مشخصات دموكرافيك افراد شركت كننده در مطالعه

\begin{tabular}{|c|c|c|c|}
\hline Pارش & 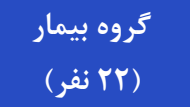 & 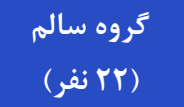 & متغير \\
\hline .1 .90 & $r \Lambda / \Lambda \subseteq \pm \Delta / \cdot Y$ & $r \Delta / \varepsilon \wedge \pm \xi / \cdot r$ & سن (سال) \\
\hline$\cdot 10 \cdot 9$ & $1 / V \cdot \pm \cdot / V$ & $1 / V T \pm \cdot / \Lambda$ & قد (متر) \\
\hline - Ifte & $V / / F \pm V / \Delta V$ & $99 / 1 \wedge \pm \wedge / \wedge \varphi$ & وزن ( كيلوكرم) \\
\hline .1 .94 & $T F / T H \pm 1 / 99$ & $r r / r \Delta \pm r / \Lambda$ & شاخص توده بدنى \\
\hline $1 / .$. & $\begin{array}{l}\mid \wedge(\Lambda \mid / \Lambda) \\
\mathcal{F}(\mid \Lambda / r)\end{array}$ & $\begin{array}{l}\mid \wedge(\Lambda \mid / \Lambda) \\
f(\mid \Lambda / T)\end{array}$ & زنسيت تعداد (درصد) \\
\hline$\cdot / \pi 91$ & $V / \cdot 9 \pm \cdot / 9$ & $V / F T \pm I / \Lambda$ & سطح فعاليت (نمره يرسشنامه بك) \\
\hline
\end{tabular}

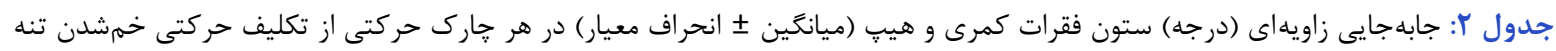

\begin{tabular}{|c|c|c|c|}
\hline 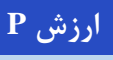 & ترو تره بيمار & كروه سالم & جارى \\
\hline \multicolumn{4}{|c|}{ زوايه خمشدن ستون فقرات كمرى } \\
\hline$\cdot 1 \cdot \sqrt{ } 9$ & $N / \Lambda \mid \pm I / F F$ & $q / F F \pm \cdot / V T$ & اول \\
\hline $.1 \cdot 19$ & $19 / 9 \cdot \pm \cdot / 9$ & $1 N / 9 r \pm 1 / 09$ & دوم \\
\hline . /Fru & $q / K F \pm \cdot / V r$ & $|r / \Lambda| \pm 1 / 99$ & سوم \\
\hline$\cdot 1 \cdot v$ & $\varphi / R \Delta \pm \cdot / \Lambda \varphi$ & $\Delta / 1 T \pm T / \cdot r$ & 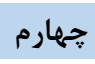 \\
\hline \multicolumn{4}{|c|}{ زاويه خمشدن هيب } \\
\hline$\cdot / \cdot V \Delta$ & $\mathrm{V} / 19 \pm \cdot / \mathrm{V} \Lambda$ & $9 / V T \pm \cdot \mid 9 F$ & اول \\
\hline$\cdot 1 \cdot 11$ & $19 / 99 \pm \cdot / 14$ & $r \cdot / r \Delta \pm \cdot / \wedge q$ & دوم \\
\hline$\cdot / \cdot \vee 1$ & $19 / T F \pm 1 / .9$ & 19/V9 $\pm \cdot / V r$ & سوم \\
\hline$\cdot 1 \cdot \mathrm{VI}$ & $9 / \wedge \Delta \pm 1 / .9$ & $q / T F \pm \cdot / V r$ & جهارم \\
\hline
\end{tabular}

دادهها بهصورت ميانخين 土 انحراف معيار براى متغييرهاى كمّى تعريف شدهاند.

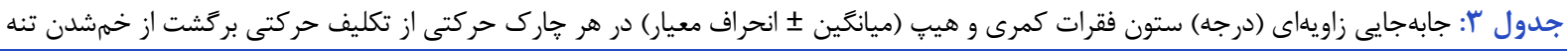

\begin{tabular}{|c|c|c|c|}
\hline ارزش & تروه بيمار & تروه سالم & 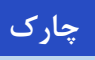 \\
\hline \multicolumn{4}{|c|}{ زوايه خمشدن ستون فقرات كمرى } \\
\hline - IVAF & $\varepsilon / 0 \cdot \pm 1 / 94$ & $\varepsilon / \varepsilon \Lambda \pm r / r \Delta$ & اول \\
\hline.$/ 1 \vee 9$ & $r \cdot / V r \pm r / 19$ & $r r / \cdot 1 \pm r /$. & دوم \\
\hline$\cdot \mid \cdot v 1$ & $\mid g / K Y \pm 1 / \cdot 9$ & IS/A $\pm \cdot / V Y$ & سوم \\
\hline . Mrr & $F / T G \pm \cdot / \Lambda F$ & $F / 9 \vee \pm \cdot / \wedge 9$ & 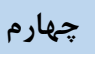 \\
\hline \multicolumn{4}{|c|}{ زاويه خمشدن هيب } \\
\hline - ITTF & $11 / 79 \pm 1 / \cdot 9$ & $11 / 4 \pi \pm \cdot / \Lambda r$ & اول - اول \\
\hline$\cdot 19 \Delta \Lambda$ & $T r / 9 \Delta \pm \cdot / V I$ & Tr/OT $\pm 1 / \cdot 9$ & 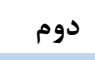 \\
\hline - /rFq & IF/AT $\pm \cdot / V G$ & $\mid F / \Delta F \pm \cdot / 19$ & 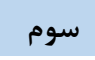 \\
\hline$\cdot|\cdot \sqrt{ }|$ & $\Delta / r \Delta \pm 1 / \cdot 9$ & $F / \Lambda F \pm \cdot / V K$ & 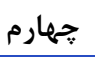 \\
\hline
\end{tabular}

دادها بهصورت ميانخين 土 انحراف معيار براى متغييرهاى كمّى تعريف شدهاند.

دوره خمشدن، ميزان ميانگين نسبت مشاركت ستون فقرات

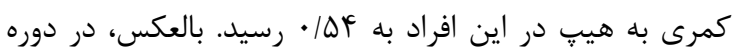

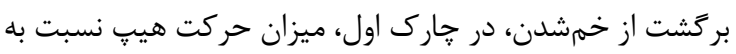

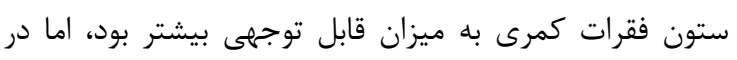

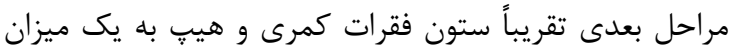

هي״ بيشتر بود و به همين خاطر، همانطور كه در جدول f أديده

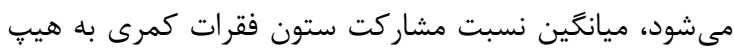

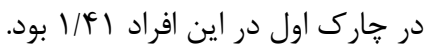

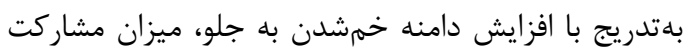

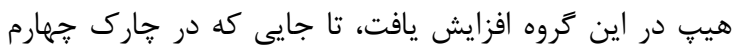




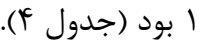

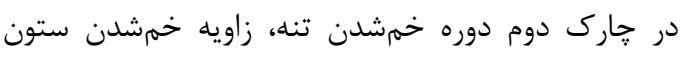

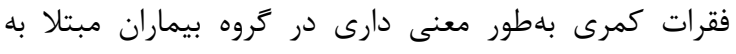

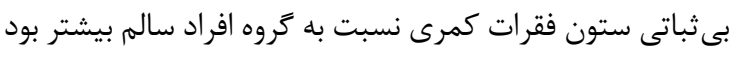

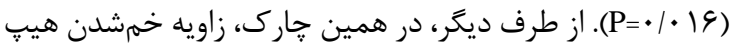

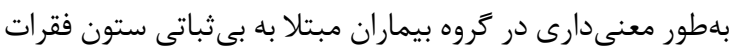

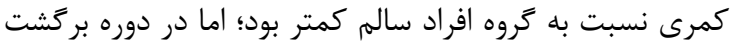

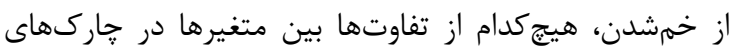

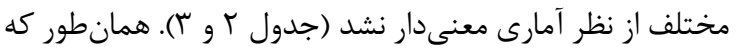

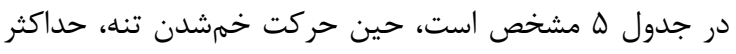

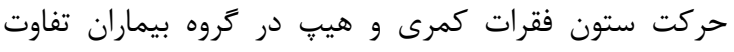

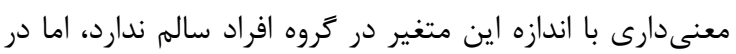

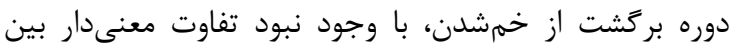

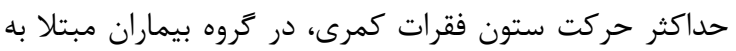

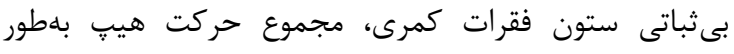

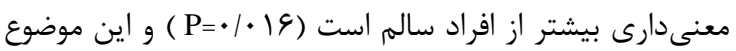

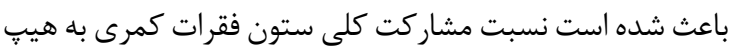

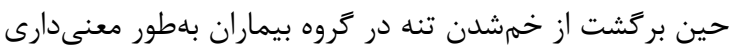

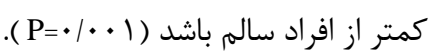

مشاركت داشتند (جدول ؟). نسبت مشاركت ستون فقرات كمرى

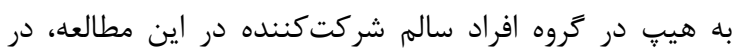

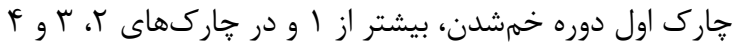

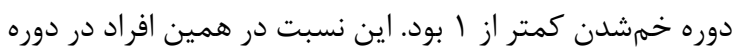

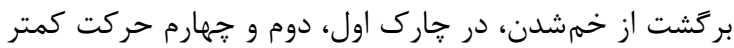

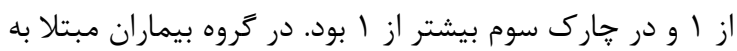

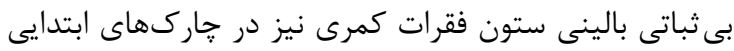

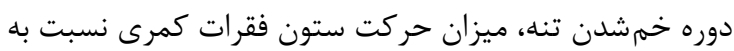

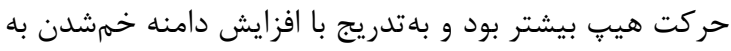

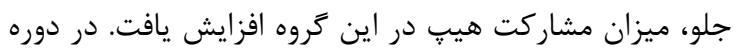

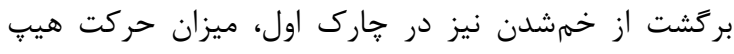

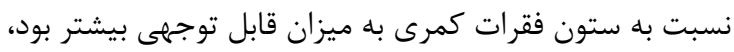

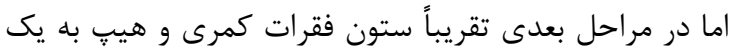

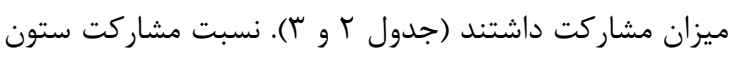

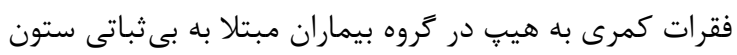

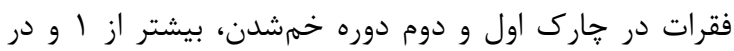

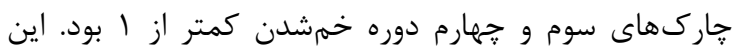

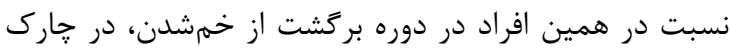

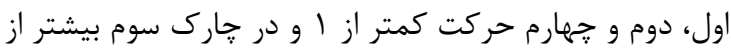

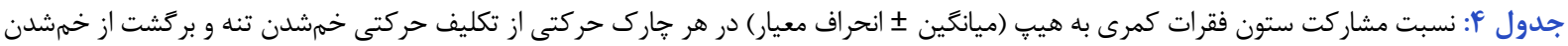

\begin{tabular}{|c|c|c|c|}
\hline ارزش & كروه بيمار & كروه سالم & جارى \\
\hline \multicolumn{4}{|c|}{ دوره خمشدن تنه } \\
\hline 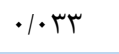 & $1 / r \Delta \pm \cdot / r q$ & $|/ F| \pm \cdot / 19$ & اول \\
\hline$\cdot 1 \cdot \cdot 1$ & $1 / \cdot 1 \pm \cdot 1 \cdot 0$ & $. / 94 \pm \cdot / \cdot 9$ & دوم \\
\hline . & $\cdot / V F \pm \cdot / \cdot \Lambda$ & $\cdot / v \cdot \pm \cdot / \cdot \Lambda$ & سوم \\
\hline.$/ . r q$ & $\cdot \mid k F \pm \cdot / 1$. & $\cdot / Q F \pm \cdot / T$. & 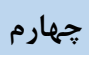 \\
\hline \multicolumn{4}{|c|}{ دوره بركشت از خمشدن تنه } \\
\hline$\cdot|99|$ & $\cdot / \Delta \omega \pm \cdot / \mid f$ & $\cdot / \Delta \Lambda \pm \cdot / r \Delta$ & 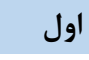 \\
\hline$\cdot / 1 \Delta$ & $\cdot 191 \pm \cdot / 14$ & $\cdot / 91 \pm \cdot / 10$ & دوم \\
\hline$\cdot|\cdot r|$ & $1 / 1 \cdot \pm \cdot / \cdot 1$ & $1 / 19 \pm \cdot 1 \cdot 1$ & سوم \\
\hline$\cdot / \cdot r \Lambda$ & . $\mid A T \pm \cdot / T K$ & $\cdot / 99 \pm \cdot / 7 \wedge$ & جهارم \\
\hline
\end{tabular}

دادهها بهصورت ميانخين I انحراف معيار براى متغييرهاى كمّى تعريف شدهاند.

جدول ه: مجموع جابهجايى زاويهاى ستون فقرات كمرى و هيب (درجه) و نسبت مشاركت ستون فقرات كمرى به هيب (ميانكين 土ـ انحراف معيار) حين

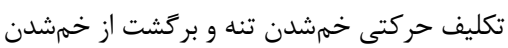

\begin{tabular}{|c|c|c|c|}
\hline ارزش & تروه بيمار & كروه سالم & \\
\hline \multicolumn{4}{|c|}{ 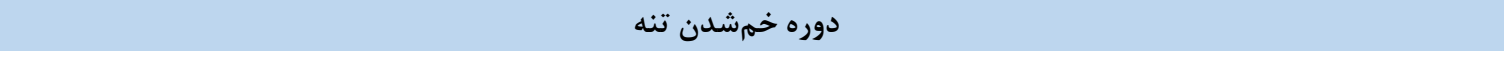 } \\
\hline$\cdot \mid \Lambda \Lambda F$ & $F V / I V \pm r / F \Lambda$ & $F V / r \cdot \pm r / T \Delta$ & زاويه خمشدن ستون فقرات كمرى \\
\hline$\cdot / F \vee G$ & $\Delta \Delta / q r \pm 1 / F$ & $09 / 19 \pm 1 / .9$ & زاويه خمشدن هيبٍ \\
\hline.$/ 914$ & $\cdot \mid \lambda F \pm \cdot / \cdot \Delta$ & $\cdot / \mathrm{AF} \pm \cdot / \cdot 9$ & نسبت مشاركت ستون فقرات كمرى به هيب \\
\hline \multicolumn{4}{|c|}{ دوره بر مشت از خمشدن } \\
\hline$\cdot / \cdot v r$ & $F V / A F \pm M / G V$ & $\Delta \cdot / 1 \Delta \pm f / \Delta \varphi$ & زاويه خمشدن ستون فقرات كمرى \\
\hline$\cdot / \cdot \cdot \Delta$ & $\Delta F / 9 r \pm 1 / 19$ & $\Delta r / T \Delta \pm 1 / 9 r$ & زاويه خمشدن هيب \\
\hline.$/ \cdot 1 \mathrm{~V}$ & $\cdot / \wedge \vee \pm \cdot / \cdot 9$ & $\cdot / 94 \pm \cdot / 1$ & نسبت مشاركت ستون فقرات كمرى به هيب \\
\hline
\end{tabular}

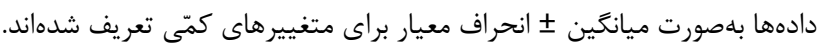


رباطها، بهعنوان عوامل اصلى كنترل ناحيه خننى نام برده مىشد؛

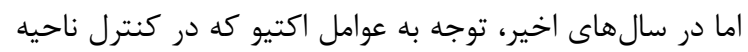

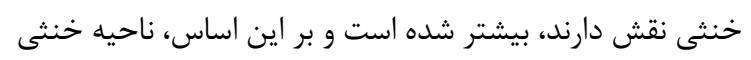

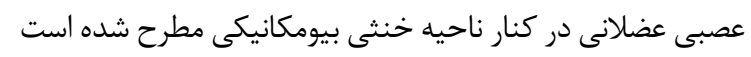

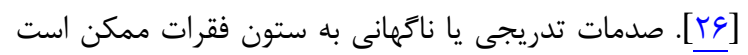

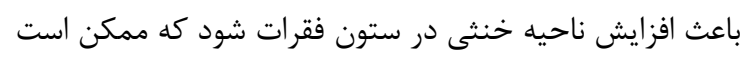

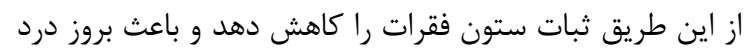

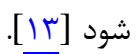

در بيماران مبتلا به بىثباتى ستون فقرات كمرى به نظر

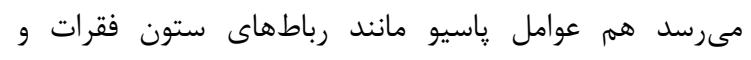
ديسكهاى بينمهرهاى و هم عوامل اكتيو عصبى عضلانى دهار

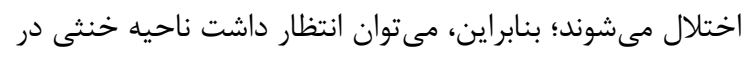

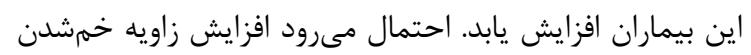

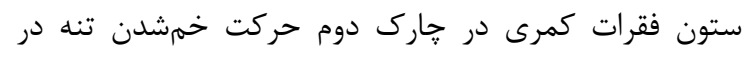

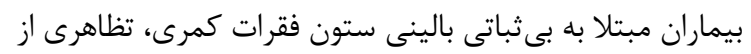

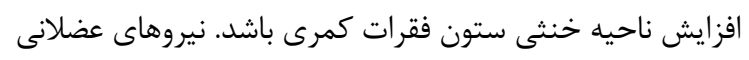

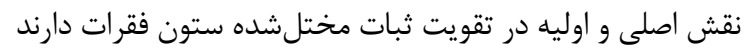

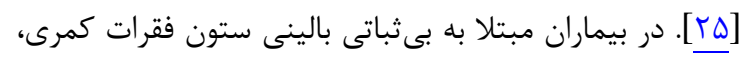

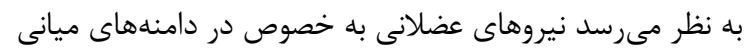

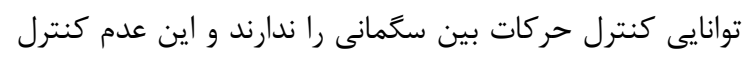

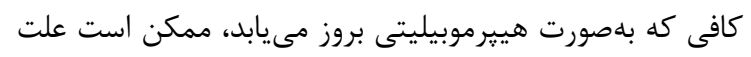

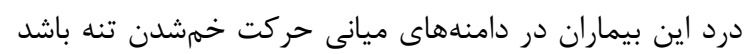

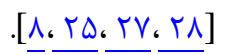

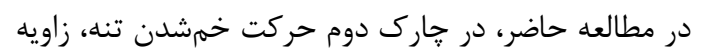

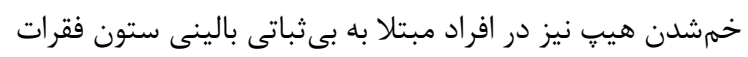

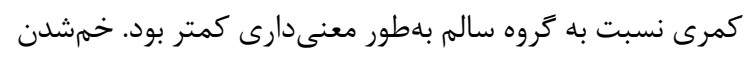

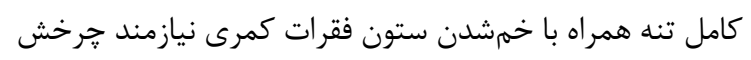

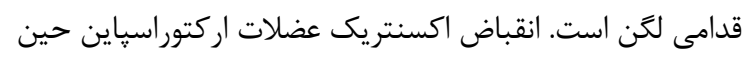

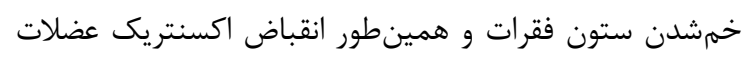

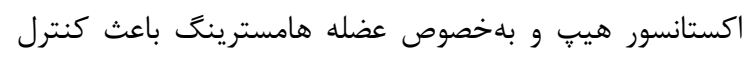

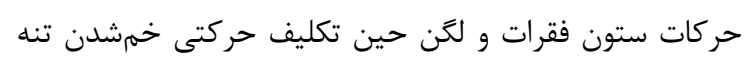

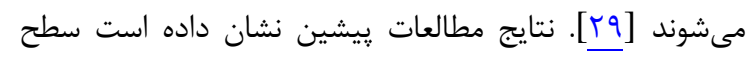

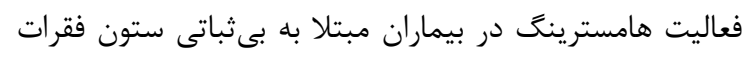

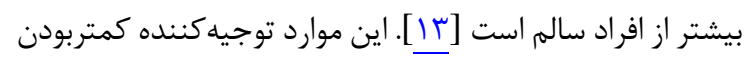

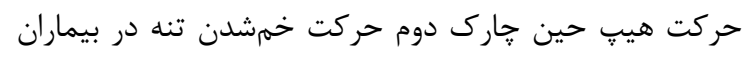

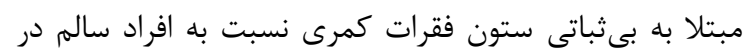

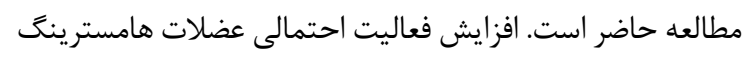

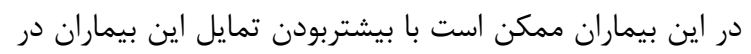

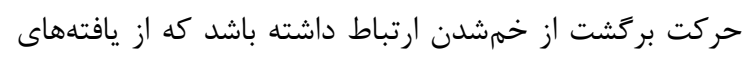
مطالعه حاضر است. تشخيص دقيق بىثباتى بالينى ستون فقرات كمرى بهواسطه

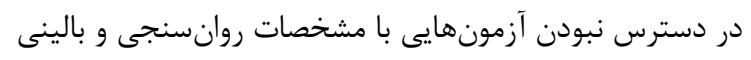

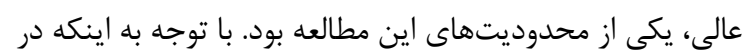

مطالعه حاضر با هدف مقايسه ريتم كمرى-لكنى در بيماران

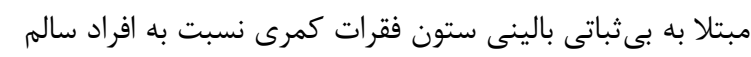

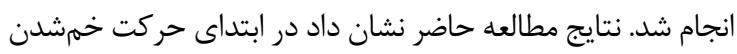

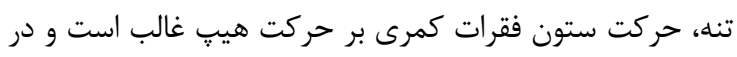

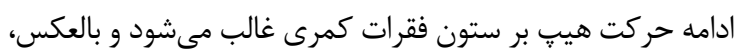

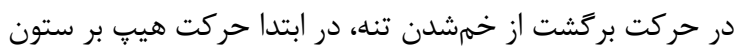

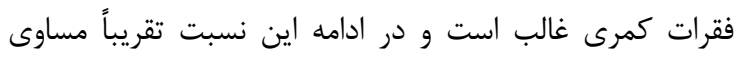

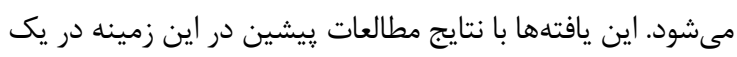

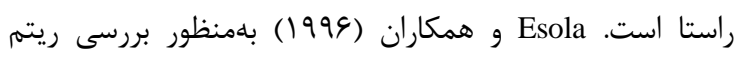

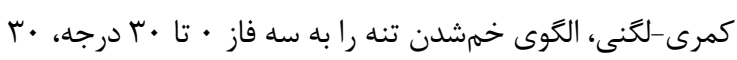

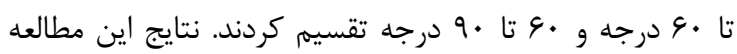

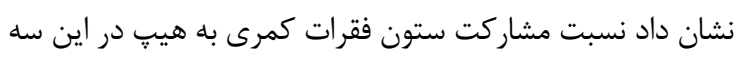

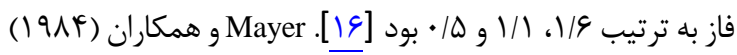

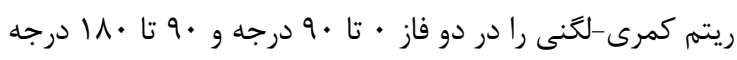

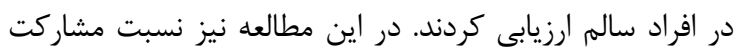

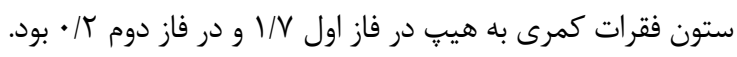

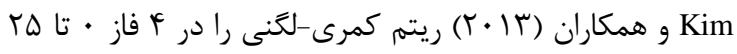

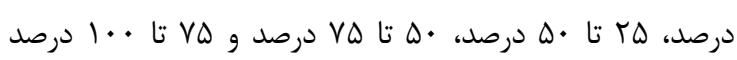

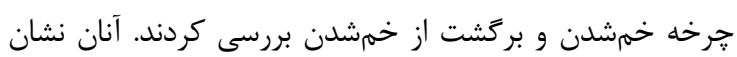

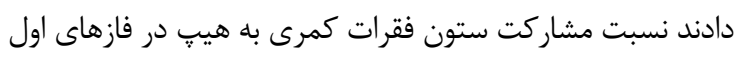

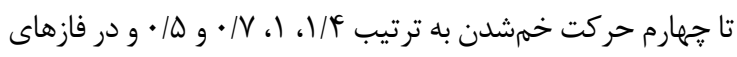

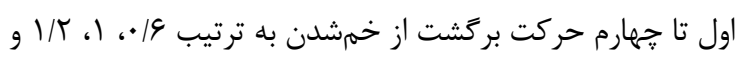

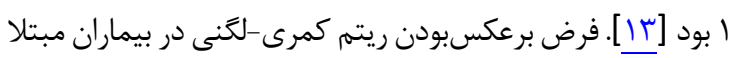

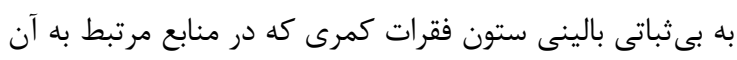

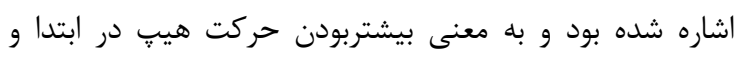

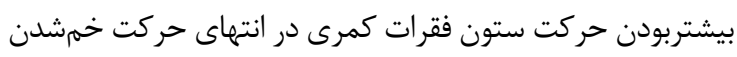

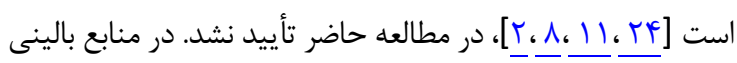

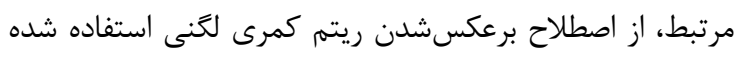

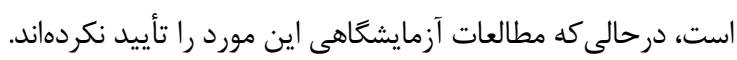

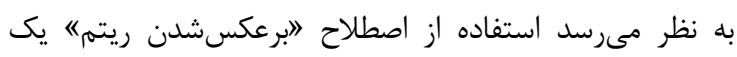

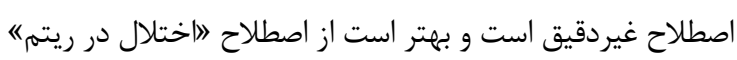

استفاده شود [1 [1].

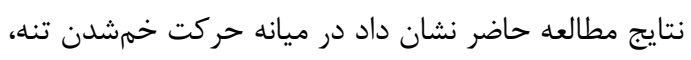

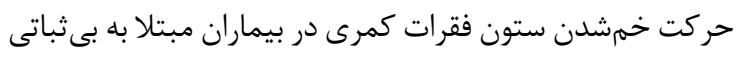

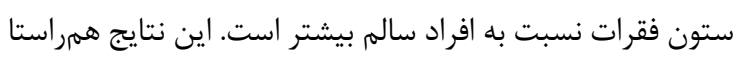

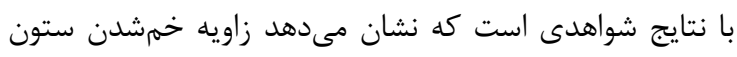

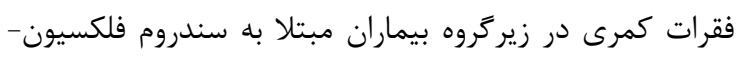

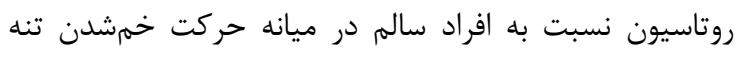

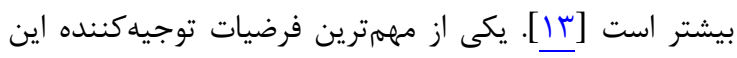

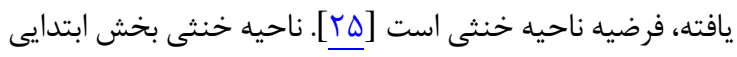

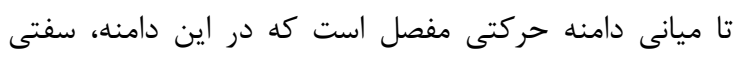

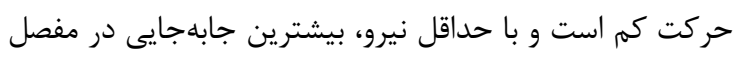

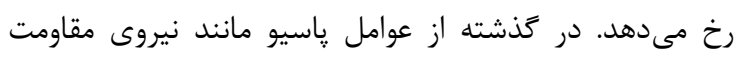


مالى براى انجام اين طرح تشكر و قدردانى مى كنند.

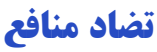

نتايج مطالعه حاضر با منافع نويسندگان در تعارض نيست.

مالاحظات اخلاقى

اين طرح از كميته اخلاق دانشخاه علوم يزشكى جندى شايور

اهواز به شناسه IR.AJUMS.REC.1394.715 تأييديه دارد.

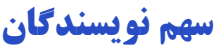

نويسنده اول (يزوهشَّر اصلى): تدوين يرويوزال، جمعآورى

دادها • ب درصد؛ نويسنده دوم (يثوهشخر اصلى): مسئول

مكاتبات، مشاركت در تدوين يرويوزال، نغارش مقاله •r درصد؛

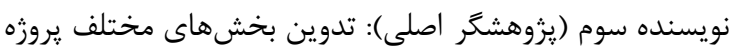

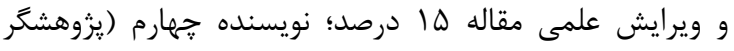

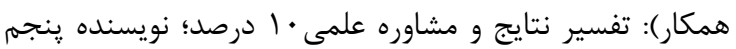

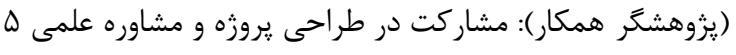

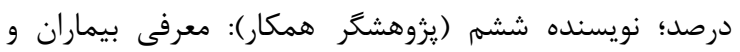

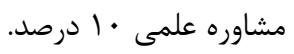

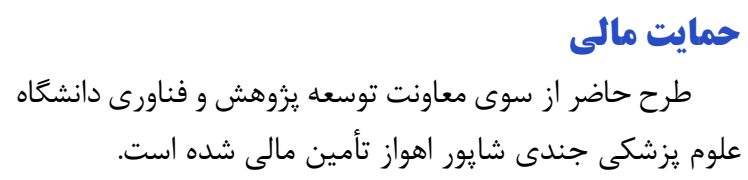

\section{REFERENCES}

1. Staal J, Hlobil H, Van Tulder M, Waddell G, Burton AK, Koes B, et al. Occupational health guidelines for the management of low back pain: an international comparison. Occup Environ Med. 2003;60(9):618-26. PMID: 12937181 DOI: $10.1136 /$ oem.60.9.618

2. Hicks GE, Fritz JM, Delitto A, Mishock J. Interrater reliability of clinical examination measures for identification of lumbar segmental instability. Arch Phys Med Rehabil. 2003;84(12):1858-64. PMID: 14669195 DOI: 10.1016/ s0003-9993(03)00365-4

3. Moissenet F, Rose-Dulcina K, Armand S, Genevay S. A systematic review of movement and muscular activity biomarkers to discriminate non-specific chronic low back pain patients from an asymptomatic population. Sci Rep. 2021;11(1):5850. PMID: 33712658 DOI: $10.1038 / \mathrm{s} 41598-$ 021-84034-X

4. Dankaerts W, O'Sullivan P, Burnett A, Straker L. Altered patterns of superficial trunk muscle activation during sitting in nonspecific chronic low back pain patients: importance of subclassification. Spine. 2006;31(17):2017-23. PMID: 16924221 DOI: 10.1097/01.brs.0000228728.11076.82

5. Hooker QL, Lanier VM, van Dillen LR. Consistent differences in lumbar spine alignment between low back pain subgroups and genders during clinical and functional activity sitting tests. Musculoskelet Sci Pract. 2021;52:102336. PMID: 33548765 DOI: 10.1016/j.msksp.2021.102336

6. Panjabi MM. Clinical spinal instability and low back pain. $J$ Electromyogr Kinesiol. 2003;13(4):371-9. PMID: 12832167 DOI: $10.1016 / \mathrm{s} 1050-6411(03) 00044-0$

7. Rathod AK, Garg BK, Sahetia VM. Lumbar rocking test: a new clinical test for predicting lumbar instability. $J$ Craniovertebr Junction Spine. 2019;10(1):33-8. PMID: 31000978 DOI: $10.4103 /$ jcvjs.JCVJS 519

8. Demoulin $\mathrm{C}$, Distree V, Tomasella M, Crielaard JM,
اين بيماران احتمال جرخش ستون فقرات كمرى حين حركت خمشدن تنه وجود دارد، ييشنههاد مى شود در مطالعات آينده متغيرهاى مرتبط با ريتم كمرى-لخنى به صورت سهبعدى و در صفحات مختلف بررسى شود. با توجه به اينكه طول عضله هامسترينگ بر حركت خمشدن تنه اثرگذار است، پيشنههاد مىشود در مطالعات آينده ارتباط طول هامسترينَ با ريتم كمرى -لخنى و يديده آسودگى خمشى بيماران مبتلا به بى ثباتى بالينى ستون فقرات كمرى مورد توجه قرار گيرد.

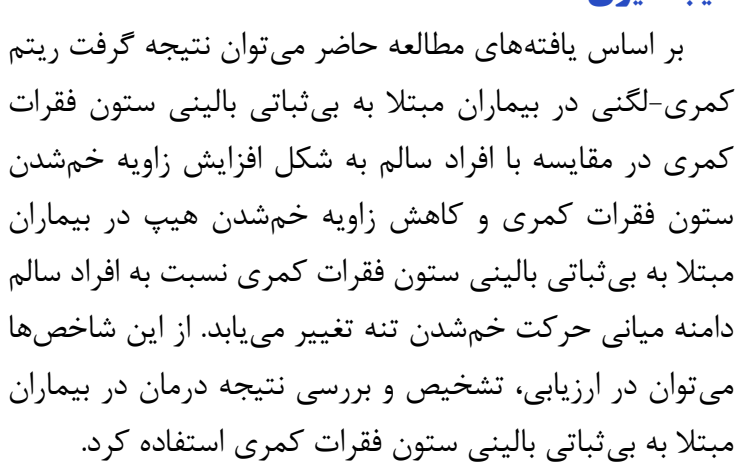
تشكر و قدر قاذى اين مقاله مستخرج از ياياننامه مصوب دانشخاه جندى شايور در مقطع دكترى تخصصى فيزيوترايى با شماره طرح pht-9435

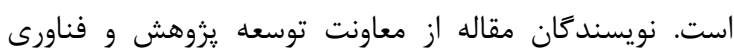
دانشگاه علوم يزشكى جندى شايور اهواز به خاطر حمايتهاى

Vanderthommen M. Lumbar functional instability: a critical appraisal of the literature. Annales de readaptation et de medecine physique. Ann Readapt Med Phys. 2007;50(8):67784. PMID: 17597247 DOI: 10.1016/j.annrmp.2007.05.007

9. Olson MW. Biomechanical characteristics of low back tissues during trunk flexion-extension. Baton Rouge, Louisiana: Louisiana State University and Agricultural \& Mechanical College; 2006

10. Watson PJ, Booker C, Main C, Chen A. Surface electromyography in the identification of chronic low back pain patients: the development of the flexion relaxation ratio. Clin Biomech. 1997;12(3):165-71. PMID: 11415689 DOI: 10.1016/s0268-0033(97)00065-x

11. Cook C, Brismée JM, Sizer PS Jr. Subjective and objective descriptors of clinical lumbar spine instability: a Delphi study. Man Ther. 2006;11(1):11-21. PMID: 15996889 DOI: 10.1016/j.math.2005.01.002

12. Alessa F, Ning X. Changes of lumbar posture and tissue loading during static trunk bending. Hum Mov Sci. 2018;57:59-68. PMID: 29161614 DOI: 10.1016/j.humov. $\underline{2017.11 .006}$

13. Kim MH, Yi CH, Kwon OY, Cho SH, Cynn HS, Kim YH, et al. Comparison of lumbopelvic rhythm and flexion-relaxation response between 2 different low back pain subtypes. Spine. 2013;38(15):1260-7. PMID: 23514875 DOI: 10.1097/ BRS.0b013e318291b502

14. Zawadka M, Skublewska-Paszkowska M, Gawda P, Lukasik E, Smolka J, Jablonski M. What factors can affect lumbopelvic flexion-extension motion in the sagittal plane? A literature review. Hum Mov Sci. 2018;58:205-18. PMID: 29482120 DOI: 10.1016/j.humov.2018.02.008

15. Vazirian M, Van Dillen L, Bazrgari B. Lumbopelvic rhythm during trunk motion in the sagittal plane: A review of the kinematic measurement methods and characterization 
approaches. Phys Ther Rehabil. 2016;3:5. PMID: 29034099 DOI: 10.7243/2055-2386-3-5

16. Hidalgo B, Gilliaux M, Poncin W, Detrembleur C. Reliability and validity of a kinematic spine model during active trunk movement in healthy subjects and patients with chronic nonspecific low back pain. J Rehabil Med. 2012;44(9):756-63. PMID: 22847223 DOI: 10.2340/16501977-1015

17. Porter JL, Wilkinson A. Lumbar-hip flexion motion: a comparative study between asymptomatic and chronic low back pain in 18-to 36-year-old men. Spine. 1997;22(13): 1508-13. PMID: 9231971 DOI: 10.1097/00007632199707010-00017

18. Esola MA, McClure PW, Fitzgerald GK, Siegler S. Analysis of lumbar spine and hip motion during forward bending in subjects with and without a history of low back pain. Spine. 1996;21(1):71-8. PMID: 9122766 DOI: 10.1097/00007632199601010-00017

19. Paquet N, Malouin F, Richards CL. Hip-spine movement interaction and muscle activation patterns during sagittal trunk movements in low back pain patients. Spine. 1994;19(5):596-603. PMID: 8184355 DOI: 10.1097/00007632-199403000-00016

20. Porter JL, Wilkinson A. Lumbar-hip flexion motion. A comparative study between asymptomatic and chronic low back pain in 18- to 36-year-old men. Spine. 1997;22(13): 1508-13. PMID: 9231971 DOI: 10.1097/00007632199707010-00017

21. Henriksen M, Lund H, Bliddal H, Danneskiold-Samsøe B. Dynamic control of the lumbopelvic complex; lack of reliability of established test procedures. Eur Spine $J$. 2007;16(6):733-40. PMID: 16957945 DOI: $10.1007 /$ s00586006-0198-5

22. Wattananon P, Ebaugh D, Biely SA, Smith SS, Hicks GE, Silfies SP. Kinematic characterization of clinically observed aberrant movement patterns in patients with non-specific low back pain: a cross-sectional study. BMC Musculoskelet Disord. 2017;18(1):455. PMID: 29141615 DOI: 10.1186/s12891-017-1820-x

23. O'Shaughnessy J, Roy JF, Descarreaux M. Changes in flexion-relaxation phenomenon and lumbo-pelvic kinematics following lumbar disc replacement surgery. J Neuroeng Rehabil. 2013;10(1):72. PMID: 23842284 DOI: 10.1186/1743-0003-10-72

24. Delitto A, Erhard RE, Bowling RW. A treatment-based classification approach to low back syndrome: identifying and staging patients for conservative treatment. Phys Ther. 1995;75(6):470-85. PMID: 7770494 DOI: $10.1093 / \mathrm{ptj} / 75.6 .470$

25. Panjabi MM. The stabilizing system of the spine. Part II. Neutral zone and instability hypothesis. J Spinal Disord. 1992;5:390-6. PMID: 1490035 DOI: 10.1097/00002517199212000-00002

26. Youssef J, Davidson B, Zhou BH, Lu Y, Patel V, Solomonow M. Neuromuscular neutral zones response to static lumbar flexion: muscular stability compensator. Clin Biomech. 2008;23(7):870-80. PMID: 18468744 DOI: $10.1016 /$ j. clinbiomech.2008.03.069

27. Ahmadi A, Maroufi N, Behtash H, Zekavat H, Parnianpour M. Kinematic analysis of dynamic lumbar motion in patients with lumbar segmental instability using digital videofluoroscopy. Eur Spine J. 2009;18(11):1677-85. PMID: 19727854 DOI: $10.1007 / \mathrm{s} 00586-009-1147-\mathrm{x}$

28. Baecke JA, Burema J, Frijters JE. A short questionnaire for the measurement of habitual physical activity in epidemiological studies. Am J Clin Nutr. 1982;36(5):936-42. PMID: 7137077 DOI: 10.1093/ajcn/36.5.936

29. van Wingerden JP, Vleeming A, Buyruk H, Raissadat K. Stabilization of the sacroiliac joint in vivo: verification of muscular contribution to force closure of the pelvis. Eur Spine J. 2004;13(3):199-205. PMID: 14986072 DOI: $10.1007 / \mathrm{s} 00586-003-0575-2$ 